\title{
Superposition of modes in a caricature of a model for morphogenesis
}

\author{
P. K. Maini \\ Department of Mathematics, University of Utah, Salt Lake City, UT 84112, USA
}

\begin{abstract}
In a model proposed for cell pattern formation by Nagorcka et al. (J. Theor. Biol. 1987) linear analysis revealed the possibility of an initially spatially uniform cell density going unstable to perturbations of two distinct spatial modes. Here we examine a simple one-dimensional caricature of their model which exhibits similar linear behaviour and present a nonlinear analysis which shows the possibility of superposition of modes subject to appropriate parameter values and initial conditions.
\end{abstract}

Key words: Mechanochemical — Superposition of modes - Cell patterning

\section{Introduction}

Recently, several models for cell patterning have been proposed based on the mechanochemical processes occurring in the early embryo. Such models have been applied to the formation of bones or of skin organ primordia, for example, feathers and scales [1-5]. These models are mathematically complex and their initial analyses has been based mainly on linear theory or numerical simulation [6]. However, recently, simple versions of the nonlinear models have been analysed [7-9]. These analyses have been carried out in parameter domains where the linear theory predicts the initial growth of a single spatial mode, and have studied the evolution of such a mode. In other parameter regimes, however, linear theory suggests that it is possible to isolate two spatial modes which may superimpose giving rise to a pattern which has two characteristic wavelengths. This has been observed in numerical simulations of one of the models $[5,10]$, proposed for scale pattern formation in the armadillo. To analyse the full nonlinear system in this case is a formidable task. Recently, such an analysis has been carried out for special simple cases of the model [10]. In this paper, we 
study a simple one-dimensional caricature model that gives similar predictions from linear analysis to the models mentioned above. In Sect. 2 we present the model and its linear analysis. In Sect. 3 we analyse the full nonlinear model for a restricted set of initial conditions and show that, depending on the initial conditions and the parameters of the model, it is possible either to get mode isolation or superposition of modes. The results of the nonlinear analysis are compared to those from numerical simulation in Sect. 4.

\section{Model and linear analysis}

The model mentioned above is a very complex system, consisting of five highly nonlinear equations. In an attempt to analyse such a system, we look at a much simpler model which captures the linear behaviour of the more complex system. The idea of setting up a simple caricature equation to study a more complicated system is not unusual (see, for example, [11]). We chose a caricature model of the form

$$
L u=-u^{p}
$$

where

$$
\begin{aligned}
& L u=\frac{\partial u}{\partial t}+\mathscr{D} u-\lambda u \\
& \mathscr{D} u=\left(\frac{\partial^{2}}{\partial x^{2}}+\alpha^{2}\right)^{2}\left(\frac{\partial^{2}}{\partial x^{2}}+\beta^{2}\right)^{2}
\end{aligned}
$$

where $x$ and $t$ are space and time respectively, $u=u(x, t), \alpha, \beta$ and $\lambda$ are real numbers (without loss of generality we take $\alpha<\beta, \alpha, \beta$ positive) and $p$ is an integer greater than 1 . The right hand side of $(2.1)$ is the simplest possible type of nonlinearity, chosen to simplify the analysis.

The spatially uniform steady states of (2.1) satisfy

$$
\left(\alpha^{4} \beta^{4}-\lambda\right) u=-u^{p}
$$

that is

$$
u=0 \text { or } u=\left(\lambda-\alpha^{4} \beta^{4}\right)^{1 /(p-1)} \text {. }
$$

We examine the linear stability of the zero steady state by considering small perturbations from $u=0$ for which nonlinear terms may be neglected. Letting

$$
u(x, t)=\hat{u}(x, t), \quad|\hat{u}(x, t)| \ll 1
$$

and substituting into (2.1) and neglecting nonlinear terms, we have the linearised equation

$$
\frac{\partial \hat{u}}{\partial t}+\mathscr{D} \hat{u}-\lambda \hat{u}=0
$$

As usual we look for solutions of the form

$$
\hat{u} \propto e^{\sigma t+i k x}
$$




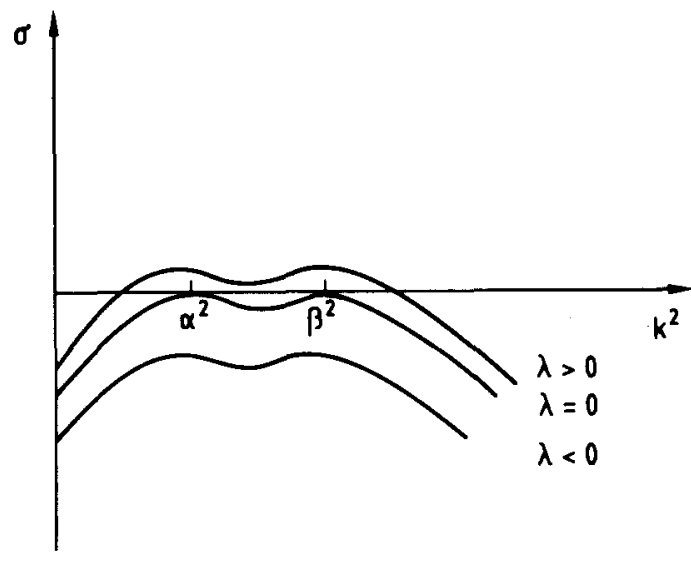

Fig. 1. Sketch of the dispersion relation (2.6) for different $\lambda$. For $\lambda<0$, $\sigma\left(k^{2}\right)<0 \forall k^{2}$ and the uniform steady state $u=0$ is linearly stable. For $\lambda=0$, $\sigma\left(k^{2}\right)=0$ at $k^{2}=\alpha^{2}, \beta^{2}$ and $u=0$ is marginally stable. For $\lambda>0, u=0$ is linearly unstable to perturbations of wave numbers in the neighbourhood of $\alpha$ or $\beta$

where $\sigma$ is the temporal growth rate of an initial perturbation of wave number $k$ (see, for example, [12]). Substituting (2.5) into (2.4), the solvability condition leads to the dispersion relation

$$
\sigma\left(k^{2}\right)=\lambda-\left(k^{2}-\alpha^{2}\right)^{2}\left(k^{2}-\beta^{2}\right)^{2}
$$

which is sketched in Fig. 1. Clearly, $\lambda$ plays the role of the bifurcation parameter in this case with $\lambda=0$ being the bifurcation value. If $\lambda<0$, then $\sigma\left(k^{2}\right)<0 \forall k^{2}$. Thus all initial perturbations die away exponentially in time and the uniform steady state $u=0$ is linearly stable. For $\lambda=0$, the uniform steady state $u=0$ is marginally stable because perturbations of wave number $\alpha$ or $\beta$ have zero linear temporal growth. If $\lambda>0$, then $\sigma\left(k^{2}\right)>0$ for two sets of wave numbers, one in the neighbourhood of $\alpha$, the other in the neighbourhood of $\beta$. Linear analysis predicts that these wave numbers will grow unboundedly. This type of linear behaviour is similar to that of the original model [5]. As these wave numbers grow, the nonlinear term in (2.1) becomes important and the linear analysis is no longer valid. We now examine nonlinear effects.

\section{The weakly nonlinear case}

We shall restrict our analysis to the case of a cubic nonlinearity, that is, $p=3$ in (2.1), and consider the case of a domain, normalised to unit length, with zero flux boundary conditions. Thus, we consider the problem

$$
\begin{gathered}
L u=-u^{3} \quad x \in(0,1) \\
\text { with } \frac{\partial^{n} u}{\partial x^{n}}(0, t)=\frac{\partial^{n} u}{\partial x^{n}}(1, t)=0 \quad \forall t, n=1,3,5,7 .
\end{gathered}
$$

To analyse the weakly nonlinear case we assume $\lambda$ is very close to its bifurcation value of 0 and use a multitime scale perturbation procedure (see, for example, [13]). In order to find the asymptotic solution in time we introduce a long time 
scale $T$. That is, we set

$$
\lambda=\epsilon^{2}, \quad T=\epsilon^{2} t, \quad u(x, t, T)=\sum_{i=1} \epsilon^{i} u_{i}(x, t, T)
$$

where $0<\epsilon \ll 1$. Substituting (3.2) into (3.1) gives

$$
L_{0}\left(\epsilon u_{1}+\epsilon^{2} u_{2}+\cdots\right)=\epsilon^{2}\left(1-\frac{\partial}{\partial T}\right)\left(\epsilon u_{1}+\epsilon^{2} u_{2}+\cdots\right)-\left(\epsilon u_{1}+\epsilon^{2} u_{2}+\cdots\right)^{3}
$$

where $L_{0} u=(\partial u / \partial t)+\mathscr{D} u$.

Equating coefficients of powers of $\epsilon$ in (3.3) leads to a hierarchy of equations to determine the functions $u_{i}$. At $O(\epsilon),(3.3)$ is

$$
L_{0} u_{1}=0
$$

with boundary conditions

$$
\frac{\partial^{n} u_{1}}{\partial x^{n}}(0, t, T)=0, \quad \frac{\partial^{n} u_{1}}{\partial x^{n}}(1, t, T)=0, \quad n=1,3,5,7
$$

The general solution of such a problem is

$$
u_{1}=\sum_{m} e^{\sigma\left(k_{m}\right) t} X\left(z, T, k_{m}\right)
$$

where $\mathscr{D} X\left(x, T, k_{m}\right)=0$ and $X$ satisfies the boundary conditions.

The linear analysis predicts that only wavelengths $\alpha$ and $\beta$ grow (at the slightly supercritical conditions considered here). We assume that

$$
\alpha=n \pi, \quad \beta=m \pi
$$

where $n$ and $m$ are integers $(n<m)$, and that we can ignore the $t$ time scale and consider temporal growth on the $T$ time scale since only modes with wavelength $\alpha$ or $\beta$ grow, and they grow slowly. To simplify the analysis further, we restrict ourselves to purely periodic disturbances (in space). Thus, the solution at $O(\epsilon)$ is

$$
u_{1}(x, T)=A(T) \cos \alpha x+B(T) \cos \beta x
$$

where $A(T)$ and $B(T)$ are functions of $T$ to be determined. The $O\left(\epsilon^{2}\right)$ terms give a similar expression for $u_{2}(x, T)$. The $O\left(\epsilon^{3}\right)$ terms are

$$
L_{0} u_{3}=u_{1}-u_{1}^{3}-\frac{\partial u_{1}}{\partial T}
$$

Secular terms arise at this stage and must be suppressed in order for $u_{3}$ to remain bounded. Note that the cubic term in (3.6) contains terms of the form $\cos 3 \alpha x$, and $\cos (2 \alpha-\beta) x$. These terms will be secular if $\beta=3 \alpha$. We consider the two cases $\beta \neq 3 \alpha, \beta=3 \alpha$.

Case $\beta \neq 3 \alpha$. In this case, the Landau equations are

$$
\begin{aligned}
& \frac{d A}{d T}=A-\frac{3}{4} A^{3}-\frac{3 A B^{2}}{2}, \\
& \frac{d B}{d T}=B-\frac{3}{4} B^{3}-\frac{3 A^{2} B}{2} .
\end{aligned}
$$




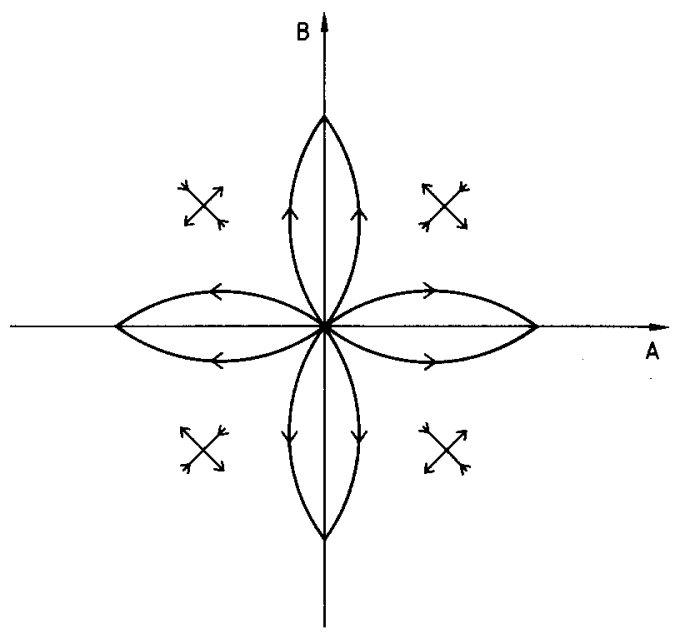

Fig. 2. Sketch of phase plane for (3.7) showing that the states $(0, \pm 2 / \sqrt{3})$ and $( \pm 2 / \sqrt{3}, 0)$ are the only stable solutions. Thus, as $T \rightarrow \infty, u_{1}(x)$ asymptotically approaches one of the forms (3.8), that is, a spatial pattern of one wavelength

This coupled system of Landau equations has steady states $(0,0),(0, \pm 2 / \sqrt{3})$, $( \pm 2 / \sqrt{3}, 0),\left( \pm \frac{2}{3}, \pm \frac{2}{3}\right)$ and $\left( \pm \frac{2}{3}, \mp \frac{2}{3}\right)$. It can easily be shown that $(0,0)$ is an unstable node, $\left( \pm \frac{2}{3}, \pm \frac{2}{3}\right)$ and $\left( \pm \frac{2}{3}, \mp \frac{2}{3}\right)$ are unstable saddle points and $(0$, $\pm 2 / \sqrt{3})$ and $( \pm 2 / \sqrt{3}, 0)$ are stable nodes. The phase plane for $(3.7)$ is sketched in Fig. 2. Clearly the solution for $u_{1}(x)$ as $T \rightarrow \infty$ can take one of the forms

$$
\pm \frac{2}{\sqrt{3}} \cos \alpha x \text { or } \pm \frac{2}{\sqrt{3}} \cos \beta x .
$$

Case $\beta=3 \alpha$. In this case, the Landau equations are

$$
\begin{gathered}
\frac{d A}{d T}=A-\frac{3}{4} A^{3}-\frac{3}{2} A B^{2}-\frac{3}{4} A^{2} B, \\
\frac{d B}{d T}=B-\frac{3}{4} B^{3}-\frac{3}{2} A^{2} B-\frac{A^{3}}{4} .
\end{gathered}
$$

This system has steady states $(0,0)$ [unstable node], $(0, \pm 2 / \sqrt{3})$ [stable node] and states $\left(A_{0}, B_{0}\right)$ where

$$
\begin{gathered}
147 A_{0}^{6}-336 A_{0}^{4}+192 A_{0}^{2}-32=0, \\
B_{0}=\left(\frac{4-7 A_{0}^{2}}{21 A_{0}^{2}-8}\right) A_{0} .
\end{gathered}
$$

Numerical solution of (3.10) gives steady states $( \pm 0.572, \mp 0.866)$ and $( \pm 0.661, \mp 0.524)$ [all saddle points] and $( \pm 1.233, \mp 0.342)$ [stable nodes]. The phase plane is sketched in Fig. 3. Clearly, in this case, the possible stable states for $u_{1}(x)$ as $T \rightarrow \infty$ in (3.10) are

$$
\pm \frac{2}{\sqrt{3}} \cos 3 \alpha, \quad \mp 1.233 \cos \alpha \pm 0.342 \cos 3 \alpha
$$

Note, that in this case, superposition of the unstable modes is possible. 


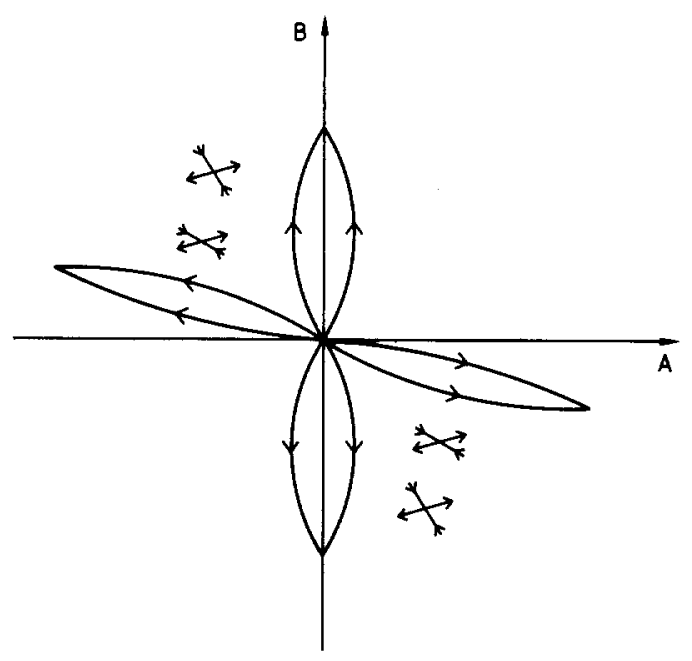

Fig. 3. Sketch of phase plane for (3.9). In this case, states $(0, \pm 2 / \sqrt{3})$ and $( \pm 1.233, \mp 0.342)$ are the stable solutions. Thus, as $T \rightarrow \infty, u_{1}(x)$ asymptotically approaches one of the forms (3.11). Clearly, depending on the initial conditions at $T=0$, it is possible for $u_{1}$ to tend to a spatial pattern with two superimposed wavelengths

\section{Numerical results}

To solve (3.1) numerically, we use the pseudo-spectral method (see [14], for example). Briefly the pseudo-spectral method approximates the solution to (3.1) by

$$
u(x, t) \simeq \sum_{j=1}^{N} a_{j}(t) \phi_{j}(x)
$$

where $\phi_{j}(x)$ are the eigenfunctions of the operator $\mathscr{D}$ defined in (2.1) satisfying the boundary conditions (3.1). Clearly

$$
\phi_{j}(x)=\cos j \pi x .
$$

Substituting for $u(x, t)$ into (3.1) we have one nonlinear equation which describes the time evolution of the amplitudes $a_{j}(t)$. We then assume that this holds at the points $x_{1}, x_{2}, \ldots, x_{n}$ (the collocation points). Thus, we now have $N$ coupled nonlinear evolution equations for the $N$ amplitudes and these are easily solved using standard ordinary differential equation solvers. Not only is this a fast and efficient method to solve such a system, it also enables us to compare the amplitudes directly with those predicted by the nonlinear analysis of Sect. 3.

We examine the two cases presented in Sect. 3.

Case $\beta \neq 3 \alpha$. In this case, if the initial conditions favour one of the unstable modes, then the solution evolves to that mode, with amplitude very close to that predicted by (3.8). However, if both unstable modes have the same initial amplitude, the solution evolves to a mixed mode, with each amplitude approximately $\frac{2}{3}$ (see Fig. 4a). In this case, the solution seems to be moving towards a saddle point in the phase plane of Fig. 2. 

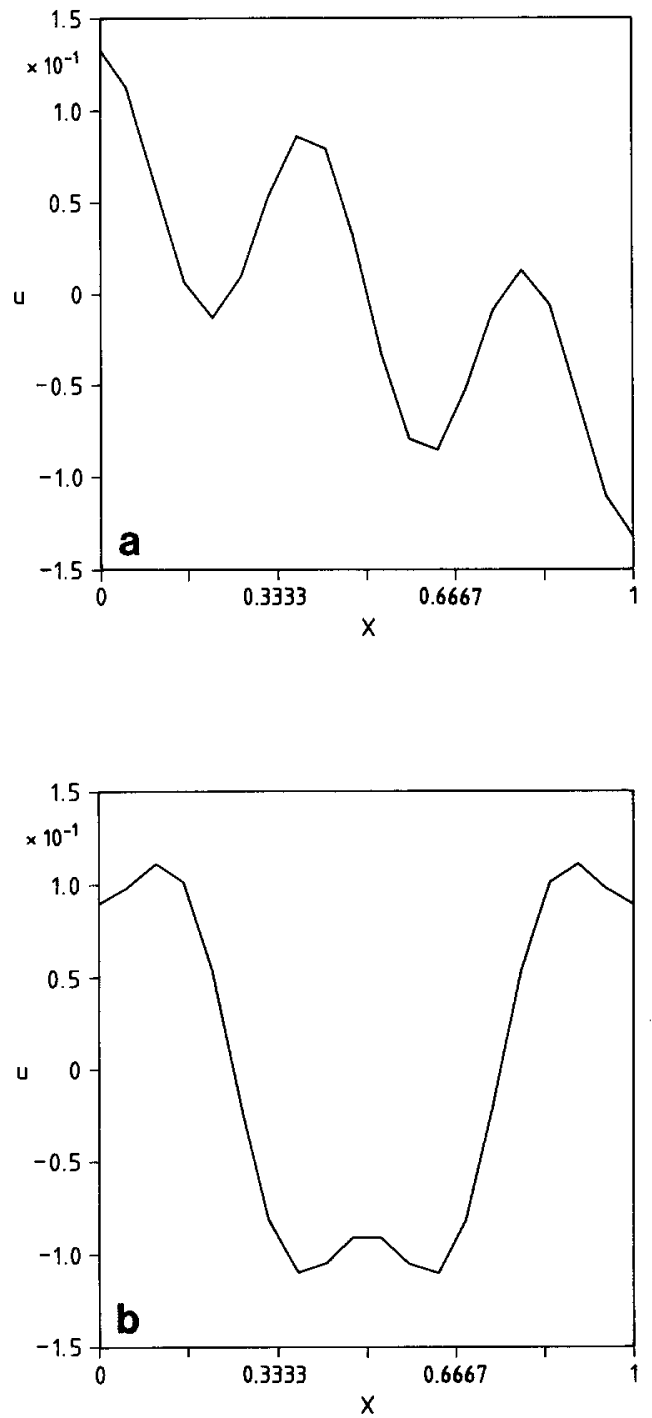

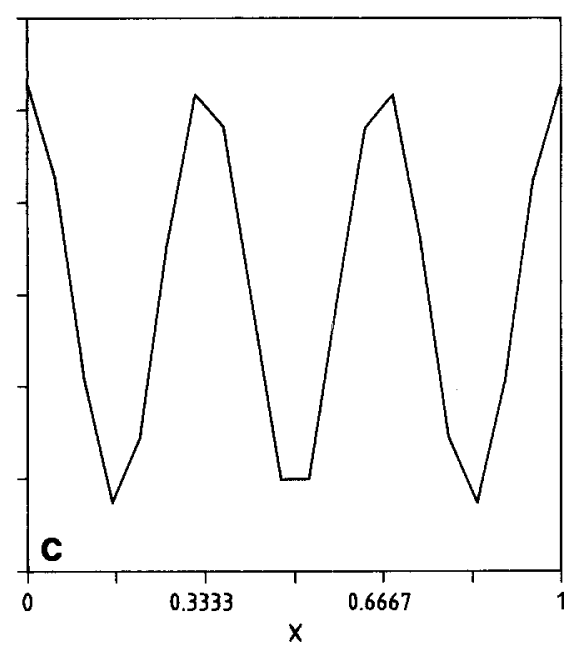

Fig. 4a-c. Numerical solution of (3.1) using the pseudo-spectral method with 20 collocation points. Initially, all modes had amplitude 0.05 .

a $\alpha=\pi, \beta=5 \pi$. In this case, all amplitudes die out very quickly, except for those of $\cos \pi x$ and $\cos 5 \pi x$. The solution converges to $0.667 \cos \pi x+0.667 \cos 5 \pi x$. This superposition of modes is qualitatively similar to that obtained in [10] for the more complex model.

b,c $\alpha=2 \pi, \beta=6 \pi$. Depending on the initial conditions, the solution either converges to a mixed mode (b), or a single mode, (c). In both cases, the results are in very good agreement with the analytic predictions of (3.11). In all the above cases, $\epsilon=0.1$

Case $\beta=3 \alpha$. In this case, the numerical solutions are in very close agreement with the nonlinear analysis. Dependent on initial conditions, the solutions evolve to either a mixed mode or a single mode, as predicted by (3.11) (Fig. 4b,c).

\section{Discussion}

Recently, several models for cell pattern formation based on mechanochemical processes have been proposed. These models exhibit very varied and complex 
behaviour. One type of behaviour that has not yet been fully studied analytically for these models is that which arises from a dispersion relation of the form sketched in Fig. 1. This predicts linear growth of two modes. This type of situation arises in fluid mechanics and the nonlinear interaction of such modes has been studied, giving rise to a phase plane similar to that in Fig. $2[15,16]$. In that case, the linearly unstable wavenumbers were consecutive. The form of the dispersion relation in Fig. 1, however, enables one to vary the sizes of the linearly unstable wave numbers independently of each other. Numerical simulation of this case in one and two dimensions shows the possibility of superposition of modes of different wavelengths $[5,10]$. This particular model, based on a cell movement system coupled to a reaction diffusion system, has been proposed as a mechanism for scale pattern formation in the armadillo. Preliminary numerical analyses shows that it predicts patterns qualitatively similar to observed scale patterns [10].

The complexity of these models makes an analytic study for such a case a formidable task. Recently, a nonlinear analysis has been carried out for the full system [10]. The analysis excludes cases where there is a special relation between the two unstable wave numbers which would give rise to extra secular terms. In this paper, in an attempt to gain some insight into the expected behaviour of such systems, we have analysed a simple caricature model giving rise to similar linear behaviour and have included the case where extra secular terms do arise. To simplify our analysis, we restricted our attention to the case of a cubic nonlinearity $(p=3)$ and to periodic spatial disturbances.

The nonlinear analysis of Sect. 3 shows that it is possible to get superposition of modes, depending on initial conditions and the relationship between the unstable modes. The results are in good agreement with numerical solutions. This analysis suggests that superposition of modes occurs only in special cases. In the original model it is possible to get superposition of modes in the case $\beta=5 \alpha$ independent of the initial conditions (see [10]) whereas, in the caricature model, this occurs only for special initial conditions (Fig. 4a). Thus, the caricature model does not capture all of the behaviour of the full system, but does enable analysis of certain cases which are formidable in the full system.

Acknowledgement. I would like to thank Annetta Cochran for typing the manuscript.

\section{References}

1. Oster, G. F., Murray, J. D., Harris, A. K.: Mechanical aspects of mesenchymal morphogenesis. J. Embryol. Exp. Morph. 78, 83-125 (1983)

2. Murray, J. D., Oster, G. F.: Cell traction models for generating pattern and form in morphogenesis. J. Math. Biol. 19, 265-279 (1984)

3. Oster, G. F., Murray, J. D., Maini, P. K.: A model for chondrogenic condensations in the developing limb: the role of extracellular matrix and cell tractions. J. Embryol. Exp. Morph. 89, 93-112 (1985)

4. Murray, J. D., Maini, P. K., Tranquillo, R. T.: Mechanochemical models for generating biological pattern and form in development. Physics Reports 171(2), 59-84 (1988) 
5. Nagorcka, B. N., Manorangan, V. S., Murray, J. D.: Complex spatial patterns from tissue interactions - an illustrative model. J. Theor. Biol. 128, 93-112 (1987)

6. Perelson, A. S., Maini, P. K., Murray, J. D., Hyman, J. M., Oster, G. F.: Nonlinear pattern selection in a mechanical model for morphogenesis. J. Math Biol. 24, 525-541 (1986)

7. Maini, P. K., Murray, J. D.: A nonlinear analysis of a mechanical model for biological pattern formation. SIAM J. Appl. Math. 48, 1064-1072 (1988)

8. Britton, N. F.: A singular dispersion relation arising in a caricature of a model for morphogensis. J. Math. Biol. 26, 387-403 (1988)

9. Maini, P. K.: Spatial and spatio-temporal patterns in a cell-haptotaxis model. J. Math. Biol. 27, 507-522 (1989)

10. Shaw, L. J., Murray, J. D.: Analysis of a model for complex skin patterns. SIAM J. Appl. Math., (in press)

11. Segel, L. A.: The structure of non-linear cellular solutions to the Boussinesq equations. J. Fluid Mech. 21, 345-358 (1965)

12. Fife, P. C.: Mathematical aspects of reacting and diffusion systems. Berlin Heidelberg New York: Springer 1977

13. Haken, H.: Synergetics. An introduction. Nonequilibrium phase transitions and self-organisation in physics, chemistry and biology. Berlin Heidelberg New York: Springer 1977

14. Eilbeck, J. C.: A collocation approach to the numerical calculation of simple gradients in reaction-diffusion systems. J. Math. Biol. 16, 233-249 (1982/83)

15. Segel, L. A.: The non-linear interaction of two disturbances in the thermal convection problem. J. Fluid Mech. 14, 97-114 (1962)

16. DiPrima, R. A., Eckhaus, W., Segel, L.A.: Non-linear wave-number interaction in near-critical two-dimensional flows. J. Fluid Mech. 49, 705-744 (1971)

Received May 24, 1989; received in revised form August 28, 1989 